\title{
Tropische Geometrie
}

Hannah Markwig

Hannah Markwig erhielt am 20. Mai den Heinz MaierLeibnitz-Preis 2010 der Deutschen Forschungsgemeinschaft (DFG). Die DFG würdigt damit die außergewöhnlichen wissenschaftlichen Leistungen der Juniorprofessorin auf dem Gebiet der Algebraischen Geometrie. In dem folgenden Beitrag stellt sie ihr Spezialgebiet, die Tropische Geometrie, kurz vor.

Tropische Geometrie, das klingt nach weißen Stränden, Palmen, Sonne und Meer. Aber die Enttäuschung gleich vorneweg: Tropische Geometrie hat eigentlich nichts mit den Tropen zu tun. Der Name wurde zu Ehren des brasilianischen Informatikers Imre Simon geprägt, der sich als einer der Ersten mit diesem Gebiet beschäftigt hat. Dennoch ist Tropische Geometrie tatsächlich so interessant wie der Name verspricht oder vielleicht sogar noch interessanter.

\section{Motivation der Tropischen Geometrie}

Die Idee der Tropischen Geometrie ist, algebraische Varietäten (also Lösungsmengen polynomialer Gleichungen) zu stückweise linearen, kombinatorischen Objekten zu degenerieren, die tropische Varietäten heißen. Trotz der Degeneration bleiben viele Eigenschaften der algebraischen Varietät erhalten. Aufgrund ihrer kombinatorischen Struktur lassen sich tropische Varietäten mit Methoden der Diskreten Mathematik untersuchen. Eine Motivation für die Tropische Geometrie ist es also, neue Methoden für die Algebraische Geometrie bereitzustellen. Besonderen Erfolg hatte die Tropische Geometrie bisher vor allem in der enumerativen Geometrie.

\section{Enumerative Geometrie}

Enumerative Geometrie ist ein Teilgebiet der Algebraischen Geometrie, in welchem man bestimmte geometrische Objekte zählt. Zum Beispiel gibt es genau eine Gerade durch zwei vorgegebene Punkte (Abbildung I).

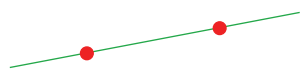

Abbildung I. Eine Gerade durch zwei Punkte

Ein weiteres Beispiel für ein enumeratives Problem ist die Frage: Wie viele Geraden im Raum treffen vier vorgegebene Geraden? Stellt man sich zwei der vier vorgegebenen Geraden gekreuzt auf dem Fußboden vor und die übrigen zwei gekreuzt an der Wand, so sieht man, dass genau zwei Geraden alle vier vorgegebenen treffen: die Gerade, die die beiden Schnittpunkte der Kreuze verbindet, und die Fußleiste (Abbildung 2).

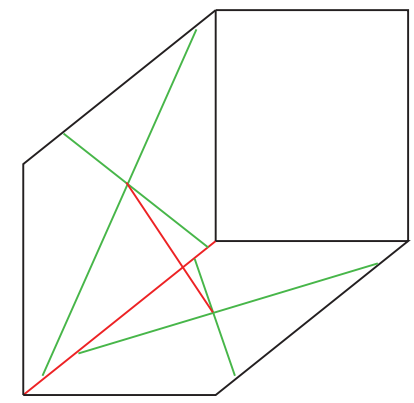

Abbildung 2. Zwei rote Geraden schneiden vier grüne Geraden im Raum

Enumerative Fragestellungen sind sehr alt. Trotzdem blieben viele solcher Fragen bis heute unbeantwortet. Eine Renaissance erlebte die enumerative Geometrie Ende der neunziger Jahre, nachdem Physiker mehrere enumerative Zahlen mit Hilfe der Stringtheorie voraussagten. Motiviert durch die Physik entwickelte sich eine neue mathematische Theorie, die Gromov-Witten-Theorie. Mit Hilfe der Gromov-Witten-Theorie konnten einige seit Langem unbekannte enumerative Zahlen gefunden werden; beispielsweise entwickelte Maxim Kontsevich die Kontsevich-Formel zur Berechnung der Zahlen rationaler ebener Kurven vom Grad $d$ durch $3 d-1$ Punkte in allgemeiner Lage. Die Tropische Geometrie stellt jetzt neue Methoden zum Studium der enumerativen Geometrie und der Gromov-Witten-Theorie bereit.

\section{Tropische Geometrie}

\section{I Tropische Rechenoperationen}

In der Tropischen Mathematik ersetzen wir zunächst die gewohnten Rechenoperationen durch neue, tropische Operationen. Statt der Operation Plus (+) verwendet man das tropische $\oplus$. Dieses Zeichen steht für die Operation Maximum. Das heißt zum Beispiel, dass $2 \oplus 3=3$ ist. Statt Mal (.) wird im Tropischen $\odot$ verwendet, das für Plus steht. Also ist $5 \odot 2=7$. Das Distributivgesetz $(a \cdot(b+c)=a \cdot b+a \cdot c)$ gilt tropisch immer noch:

$$
\begin{aligned}
a \odot(b \oplus c) & =a+\max \{b, c\} \\
& =\max \{a+b, a+c\} \\
& =a \odot b \oplus a \odot c
\end{aligned}
$$


Andere Formeln gelten aber nicht mehr oder in abgewandelter Weise. So wird die binomische Formel $(a+$ b) $\cdot(a+b)=a \cdot a+2 \cdot a \cdot b+b \cdot b$ in der tropischen Welt viel einfacher, denn tropisch gilt:

$$
\begin{aligned}
(a \oplus b) \odot(a \oplus b) & =2 \cdot \max \{a, b\} \\
& =\max \{2 a, 2 b\} \\
& =a \odot a \oplus b \odot b
\end{aligned}
$$

\subsection{Tropische ebene Kurven}

In der Algebraischen Geometrie ist eine Gerade durch eine lineare Gleichung gegeben. Wählen wir zum Beispiel die Gerade $x+y+1=0$. Wenn wir die linke Seite nun tropikalisieren (also + durch $\oplus$ und · durch $\odot$ ersetzen), so erhalten wir $\max \{x, y, 1\}$. Tropisch ergibt das GleichNull-Setzen keinen wirklichen Sinn, da die übliche Null tropisch kein neutrales Element ist. Tropisch gesehen ist zum Beispiel $-3 \oplus 0=\max \{-3,0\}=0 \neq-3$. (Das additive Neutrale ist $-\infty .(\mathbb{R} \cup\{-\infty\}, \oplus, \odot)$ ist ein Semiring, denn es gibt keine additiven Inversen.) Anstatt die linke Seite Null zu setzen, betrachten wir all die Punkte $(x, y)$ der Ebene, für die das Maximum $\max \{x, y, 1\}$ nicht eindeutig ist, also von mindestens zwei Termen angenommen wird. (Eine Erklärung für die Wahl dieser Alternative zum Gleich-Null-Setzen ergibt sich, wenn man tropische Kurven als Degenerationen algebraischer Kurven auffasst.) Dies wird zum Beispiel von allen Punkten $(x, y)$ erfüllt, für die $x=y \geq 1$ ist, also für eine halbe Diagonale, die am Punkt $(1,1)$ beginnt. Außerdem ist die Bedingung von allen Punkten erfüllt, für die $x=1 \geq y$ oder $y=1 \geq x$ ist. Dies sind zwei Halbgeraden, die parallel zu den Achsen sind und sich im Punkt $(1,1)$ treffen. Die tropische Gerade (bestehend aus drei Halbgeraden), die zu $\max \{x, y, 1\}$ gehört, sieht also so aus:

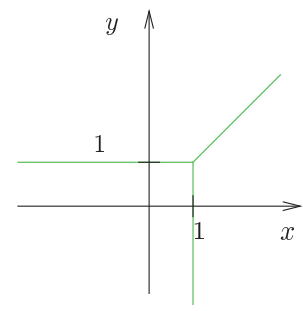

Abbildung 3. Die tropische Gerade $x \oplus y \oplus 1$

Wiederholt man das Spiel mit einer Gleichung vom Grad zwei oder drei, so erhält man Bilder wie in den Abbildungen 4 und 5 .

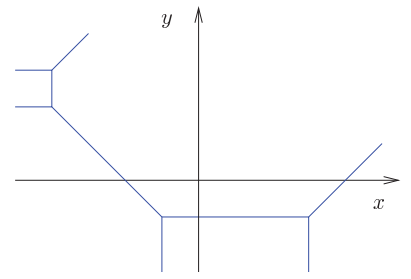

Abbildung 4. Die tropische Parabel

$3 \oplus 4 \odot x \oplus 1 \odot x \odot x \oplus 1 \odot y \oplus 5 \odot x \odot y \oplus-2 \odot y \odot y$



Abbildung 5. Die tropische Kubik

$1 \oplus 5 / 2 \odot y \oplus 3 \odot y \odot y \oplus 1 \odot y \odot y \odot y \oplus 4 \odot x \oplus-2 \odot x \odot$ $y \oplus 9 \odot y \odot y \odot x \oplus 2 \odot x \odot x \oplus 7 \odot y \odot x \odot x \oplus-1 \odot x \odot x \odot x$

Natürlich gibt es in der Tropischen Geometrie nicht nur Kurven in der Ebene, sondern auch höherdimensionale Objekte. Abbildung 6 zeigt zum Beispiel eine tropische Ebene im Raum.

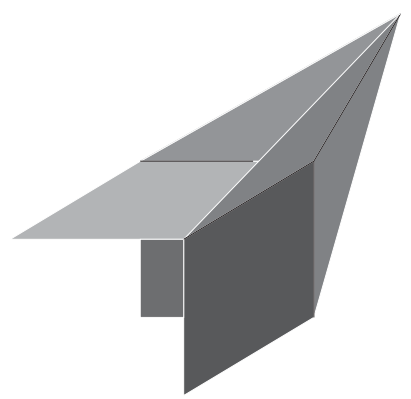

Abbildung 6. Die tropische Ebene $x \oplus y \oplus z \oplus 1$

\section{Tropische enumerative Geometrie}

Die enumerativen Fragen, die wir oben gestellt haben, können wir ebenso auch tropisch stellen, zum Beispiel: Wie viele tropische Geraden gehen durch zwei Punkte? Abbildung 7 zeigt, dass es wie erwartet genau eine tropische Gerade ist.

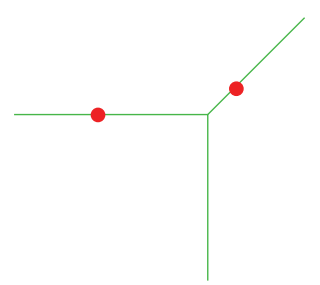

Abbildung 7. Eine tropische Gerade durch zwei Punkte

Natürlich können auch kompliziertere enumerative Fragen mit Hilfe der Tropischen Geometrie beantwortet werden. Abbildung 8 zeigt zum Beispiel, dass es zwölf (rationale) Kubiken (also Kurven vom Grad 3) durch acht vorgegebene Punkte gibt. 




Abbildung 8. Zwölf rationale tropische Kubiken durch acht Punkte

(Dabei muss die Kurve unten rechts vierfach gezählt werden, was daran liegt, dass mehrere algebraische Kubiken zur selben tropischen Kubik degenerieren.)

Mit Hilfe der Tropischen Geometrie konnten verschiedene enumerative Zahlen bestimmt werden, die vorher nicht bekannt waren. Besonders die Berechnung der Welschinger-Invarianten durch Grigory Mikhalkin im Jahr 2003 hat für großes Aufsehen gesorgt. Es gibt aber auch noch viele offene Fragen in diesem Bereich. So ist zum Beispiel die Frage, wie viele tropische Geraden auf einer tropischen Fläche im Raum zu finden sind, nur teilweise beantwortet.

Gemeinsam mit Andreas Gathmann habe ich einen tropischen Beweis der oben erwähnten Konsevich-Formel entwickelt. Auch andere bekannte Algorithmen zur Bestimmung enumerativer Zahlen haben wir in der tropischen Welt wiedergefunden, so zum Beispiel den Caporaso-Harris-Algorithmus, dessen Idee im folgenden Kapitel beschrieben wird. Die neuen Methoden erlauben Verallgemeinerungen, die ohne den tropischen Ansatz nicht möglich wären. Zum Beispiel habe ich gemeinsam mit Michael Kerber eine neue Formel für bestimmte Zahlen ebener rationaler Kurven gefunden. Ilia Itenberg, Viatcheslav Kharlamov und Eugenii Shustin haben unsere Methoden weiterentwickelt, um bessere Algorithmen für Welschinger-Invarianten zu finden. Es gibt noch viele Fragen der enumerativen Geometrie, für die man sich Antworten mit tropischen Methoden erhofft. Das setzt natürlich ein intensives Studium der tropischen enumerativen Geometrie selbst voraus, mit deren Entwicklung ich mich schwerpunktmäßig beschäftige. Dabei werden viele Methoden analog zu Methoden der klassischen enumerativen Geometrie entwickelt (wobei das Wort „klassisch“ hier im Unterschied zu tropisch verstanden werden soll). Klassisch lassen sich viele enumerative Zahlen als Schnittprodukte auf einem geeigneten Modulraum darstellen. Dabei wählt man einen Modulraum, der die Objekte, die man zählen möchte, parametrisiert - beispielsweise den Raum der Geraden in der Ebene. Jede Bedingung, die man stellt - zum Beispiel die Bedingung, durch einen bestimmten Punkt zu gehen - wird von Kurven erfüllt, die durch einen Unterraum des Modulraums parametrisiert werden. Um Kurven zu erhalten, die alle Bedingungen erfüllen, muss man dann nur noch alle Unterräume schneiden und die Punkte im Schnittprodukt zählen. Zur Zeit beschäftige ich mich eingehend mit der Konstruktion von neuen tropischen Modulräumen von Kurven, mit der Definition von tropischen enumerativen Zahlen als tropische Schnittprodukte auf diesen Modulräumen und mit Anwendungen der tropischen enumerativen Geometrie in der klassischen Welt.

\section{Der Caporaso-Harris-Algorithmus und sein} tropisches Analogon

Lucia Caporaso und Joe Harris haben 1998 einen Algorithmus entwickelt, mit dem sich die Zahlen ebener Kurven von gegebenem Grad und Geschlecht durch Punkte erstmals bestimmen ließen. Ihre Strategie ist es, zunächst allgemeinere Zahlen von Kurven zu definieren, nämlich solche, die neben Punktbedingungen auch Tangentialbedingungen an eine Gerade $L$ erfüllen. Indem ein Punkt nach dem anderen auf die Gerade geschoben wird - also, indem die Lage der Punkte spezialisiert wird - werden rekursive Formeln für diese Zahlen hergeleitet. Mit diesen Formeln kann man auch die Zahlen von Kurven berechnen, die ausschließlich Punktbedingungen erfüllen.

Als Beispiel für diesen Spezialisierungsprozess betrachten wir ebene rationale Kubiken, die Kontaktordnung 3 an $L$ in einem festen Punkt $p_{1}$ haben und außerdem durch fünf allgemeine Punkte $p_{2}, \ldots, p_{6}$ gehen. Um diese Zahl zu berechnen, bewegen wir den Punkt $p_{2}$, bis er auf $L$ liegt. Was passiert mit den Kubiken bei dieser Spezialisierung? Da sie vorher $L$ bereits mit Vielfachheit 3 in $p_{1}$ geschnitten haben, können sie nicht durch einen weiteren Punkt auf $L$ gehen, es sei denn, sie werden reduzibel und $L$ spaltet sich als Komponente ab. Es gibt zwei Möglichkeiten, wie das passieren kann: Die Kubiken können zu einer Vereinigung von drei Geraden $L \cup L_{1} \cup L_{2}$ degenerieren, wobei $L_{1}$ und $L_{2}$ jeweils durch zwei der Punkte $p_{3}, \ldots, p_{6}$ gehen. Oder sie können zu einer Vereinigung $L \cup C$ degenerieren, wobei $C$ eine Konik ist, die tangential an $L$ ist und durch $p_{3}, \ldots, p_{6}$ geht (siehe Abbildung 9).

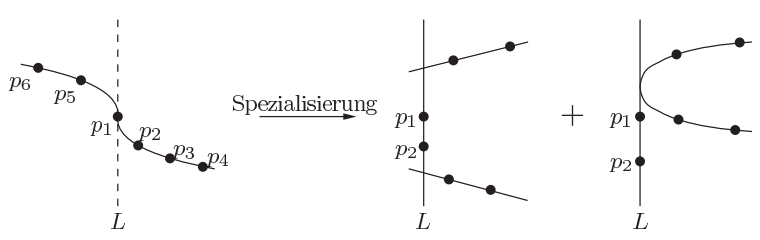

Abbildung 9. Die Caporaso-Harris Spezialisierung bei Kubiken

Die ursprüngliche Zahl von Kubiken mit einem Punkt von Kontaktordnung 3 und durch fünf weitere Punkte ist daher eine Summe von zwei Zahlen von Koniken bzw. Geraden (mit passender Multiplizität gezählt). So wird der 
Grad der Kurven reduziert, und wir erhalten durch die Spezialisierung eine rekursive Formel.

Im Tropischen entspricht eine höhere Kontaktordnung einer Situation, in der die unbeschänkten Kanten nach links (siehe Abbildung 5) zusammenschmelzen zu einer Kante von höherem Gewicht. Die Kubiken mit einem Punkt von Kontaktordnung 3 entsprechen tropischen Kubiken, die eine unbeschränkte Kante von Gewicht drei nach links haben (und außerdem durch fünf Punkte gehen). Der Spezialisierungsprozess besteht darin, den Punkt $p_{2}$ weit nach links zu schieben (siehe Abbildung 10).

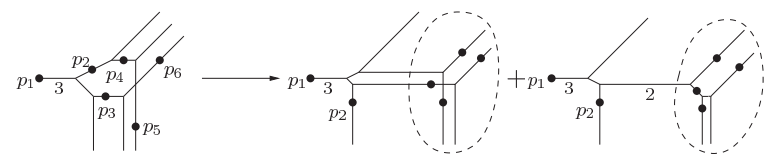

Abbildung 10. Die Caporaso-Harris-Spezialisierung bei tropischen Kubiken

Die dadurch entstehenden tropischen Kurven spalten sich in zwei Teile: den linken Teil (durch $p_{2}$ ) und den rechten Teil durch die übrigen Punkte (im Bild 10 umkreist). Wir erhalten dieselben Degenerationen wie im klassischen Fall: eine, bei der der rechte Teil aus zwei Geraden durch jeweils zwei der Punkte $p_{3}, \ldots, p_{6}$ besteht, und eine, bei der er aus einer Konik mit einer linken unbeschränkten Kante von Gewicht zwei besteht, also einer Konik, die tangential ist.

Das Beispiel des Caporaso-Harris-Algorithmus zeigt, wie gut sich Konzepte aus der enumerativen Geometrie in die tropische Geometrie übersetzen lassen. Dadurch wird deutlich, wie vielversprechend der neue tropische Ansatz für die enumerative Geometrie ist.

Prof. Dr. Hannah Markwig, CRC Higher Order Structures for Mathematics, Mathematisches Institut, Georg August Universität Göttingen, Bunsenstr. 3-5, 37073 Göttingen. hannah@uni-math.gwdg. de

Juniorprofessorin Dr. Hannah Markwig hat von 1999-2003 in Kaiserslautern Mathematik mit den Nebenfächern Physik und Philosophie studiert (Auslandssemester in Berkeley 2002) und von 2003-2006 in Kaiserslautern bei Andreas Gathmann promoviert. 2006-2007 war sie als Postdoc am IMA (Institute for Mathematics and its Applications) in Minneapolis und 2007-2008 Postdoc Assistant Professor an der University of Michigan, Ann Arbor. Seit 2008 ist sie Juniorprofessorin in Göttingen. Hannah Markwig leitet am Courant Forschungszentrum „Strukturen höherer Ordnung in der Mathematik“ die Nachwuchsgruppe „Tropische Algebraische Geometrie“.



Am Mathematischen Forschungsinstitut Oberwolfach (MFO) ist die Stelle

\section{der Stellvertretenden Direktorin bzw. des Stellvertretenden Direktors}

neu zu besetzen.

Das MFO ist ein Institut der Leibniz-Gemeinschaft und gilt in der Mathematik international als eine der renommiertesten Forschungseinrichtungen. Jährlich arbeiten dort ca. 2500 Mathematiker im Rahmen von wöchentlichen bis dreimonatigen Forschungsaufenthalten an aktuellen Problemstellungen aus der gesamten Bandbreite der Mathematik einschließlich ihrer Anwendungen. Weitere Informationen zum MFO findet man unter www.mfo. de.

Die Aufgabe der Stellvertretenden Direktorin bzw. des Stellvertretenden Direktors besteht in der Mitarbeit bei der wissenschaftlichen und organisatorischen Leitung des Instituts. Sie bzw. er vertritt den Direktor in allen Angelegenheiten.

Das Amt wird in Nebentätigkeit mit zwanzig Prozent einer Vollzeitbeschäftigung ausgeübt. Die Amtszeit beginnt am 1.2. 2012 und beträgt 5 Jahre, eine Wiederbestellung ist möglich.

Gesucht wird eine Persönlichkeit, die in der mathematischen Forschung international ausgewiesen ist und die ihre hauptamtliche Tätigkeit möglichst in einer benachbarten Universität oder Forschungseinrichtung, auch im benachbarten Ausland, ausübt.

Bewerbungen mit den üblichen Unterlagen (einschließlich ausführlicher Lebenslauf, Schriftenverzeichnis, Forschungsinteressen, ausgewählte Veröffentlichungen) werden bis spätestens 31. Juli 2010 per Email an jaeger@mfo.de als pdf-Dateien erbeten.

Insbesondere werden Frauen mit entsprechender Qualifikation zur Bewerbung ermuntert. Schwerbehinderte werden bei gleicher Eignung vorrangig eingestellt.

Prof. Dr. Dr. h.c. mult. Willi Jäger Vorsitzender der Gesellschaft für Mathematische Forschung Institut für Angewandte Mathematik/IWR Universität Heidelberg jaeger@mfo.de 